\title{
Cambio, acción humana y desarrollo en Albert O. Hirschman: reflexiones económico-filosóficas a sesenta años de The Strategy of Economic Development ${ }^{1}$
}

\author{
Gonzalo Carrión ${ }^{2}$
}

Enviado: 22 de mayo de 2019 / Aprobado: 25 de septiembre de 2019

Resumen. Albert O. Hirschman (1915-2012) ha sido uno de los científicos sociales más ingeniosos y originales en el estudio de las causas y consecuencias, obstáculos y ventajas, marchas y contramarchas, de y hacia el progreso en los países subdesarrollados. El presente trabajo tiene dos objetivos principales. Por un lado, mostrar la fecundidad de la manera hirschmaniana de comprender y responder a diversas problemáticas económicas. Por otro, proponer como clave de lectura para su obra las reflexiones sobre la interacción entre la comprensión de la acción humana y el cambio social mediante un análisis de la denominada "trilogía del desarrollo", partiendo de The Strategy of Economic Development (1958). A través de la argumentación, se sugerirá que la lectura integral de la obra de Hirschman puede ser revalorizada y servir como inspiración para el abordaje de problemáticas actuales tanto para la economía como para el resto de las ciencias sociales.

Palabras clave: Albert O. Hirschman, acción humana, cambio social, decisiones, desarrollo

Clasificación JEL: B310, B400, O1

[en] Change, human action and development in Albert O. Hirschman: some economic philosophical considerations sixty years after The Strategy of Economic Development

Abstract. Albert O. Hirschman (1915-2012) has been one of the most ingenious and original social scientists in the study of causes and consequences, obstacles and advantages, marches and counter-marches, of and toward progress in underdeveloped countries. This paper has two main objectives. On the one hand, to show the fertility of the Hirschmanian manner of understanding and responding to various economic problems. On the other, to propose as a key to reading for his work the reflections on the interaction between the understanding of human action and social change through an analysis of the so-called "trilogy of development", starting from The Strategy of Economic Development (1958). Through this argumentation, it will be suggested that the comprehensive reading of Hirschman's work could be revalued and serve as inspiration to address current issues for both the economy and the other social sciences.

Keywords: Albert O. Hirschman, human action, social change, decisions, development.

\section{JEL classification: B310, B400, O1}

Sumario: 1 Introducción. 2. Albert O. Hirschman, pionero del desarrollo. 3. Acción humana y perspectiva de crecimiento en The Strategy of Economic Development. 4. Expansión del enfoque hirschmaniano del cambio social a las decisiones políticas. 5. Ampliación de criterios de evaluación en proyectos de desarrollo. Referencias bibliográficas

Cómo citar: Carrión, G. (2019) Cambio, acción humana y desarrollo en Albert O. Hirschman: reflexiones económicofilosóficas a sesenta años de The Strategy of Economic Development, en Iberian Journal of the History of Economic Thought 6(2) (2019), 157-174

1 Cabe aclarar que el libro de Hirschman se publicó en 1958, mientras que el presente artículo fue escrito en su mayor parte a fines del año 2018, de ahí la referencia a los sesenta años. Agradezco los comentarios y sugerencias tanto de los revisores anónimos como de los editores. Quedan, asimismo, exentos de los errores que pudieran persistir y de las ideas aquí expuestas.

2 Universidad Nacional de Villa María gcarrion@unvm.edu.ar 


\section{Introducción}

En el prólogo a la edición conmemorativa de los veinte años de Las pasiones y los intereses, Amartya Sen se refiere a Albert O. Hirschman (1915-2012) como "uno de los grandes intelectuales de nuestro tiempo", dado que sus escritos "transformaron nuestro entendimiento del desarrollo económico, las instituciones sociales, la conducta humana y la naturaleza e implicaciones de nuestras identidades, lealtades y compromisos" (1997, 10). Más cerca en el tiempo Dani Rodrik se refería a él como "una de las mentes más creativas que ha dado la economía" (2016, 152). Sin duda, Hirschman ha sido reconocido como uno de los científicos sociales que, partiendo de estudios económicos, más ha insistido en el diálogo interdisciplinario no solamente entre las ciencias sociales sino también con las ciencias humanas y la filosofía. De allí que se presente como un pensador de gran originalidad, profundidad y complejidad. No obstante, al mismo tiempo puede decirse que, en general, su obra es relativamente poco conocida, en particular entre los economistas. Más allá de algunas menciones referidas a aspectos puntuales de sus aportes en la economía del desarrollo, o algunos conceptos por él acuñados, bien podría afirmarse que la obra hirschmaniana, desde una perspectiva integral, ha quedado -injustamente- relegada dentro de la formación del economista actual.

Teniendo en cuenta lo anterior, el presente trabajo tiene dos objetivos principales intrínsecamente vinculados. Por un lado, mostrar la fecundidad de la manera hirschmaniana de comprender y responder a diversas problemáticas económicas. Por otro, proponer una clave de lectura para su obra a partir de un análisis de la denominada "trilogía del desarrollo"; conjunto de obras que, al decir de Alacevich (2017a), ha quedado relativamente eclipsada por la concentración de la crítica en los textos subsiguientes del autor. Dicha clave de lectura ubica en el núcleo de la reflexión hirschmaniana a la interacción entre la comprensión de la acción humana y el cambio social. En efecto, tratará de mostrarse que la percibida insuficiencia con que la economía ha tratado el problema del proceso de cambio social, particularmente encarnado en las experiencias latinoamericanas de mediados del siglo XX, lleva a Hirschman a tratar de arrojar luz sobre la cuestión mediante el estudio de las complejas interacciones entre las visiones-imágenes de cambio y las contradicciones y tensiones de la voluntad entre las que los agentes toman las decisiones que pueden conducir al progreso. A su vez, se intentará mostrar que dicho enfoque no sólo conlleva una concepción alternativa para la comprensión -e intervención en pos- del desarrollo económico en general, sino que reclama una reconfiguración en el entendimiento de la generación y consecuencias de las decisiones políticas de las autoridades, así como también una ampliación de los criterios para la evaluación y ejecución de proyectos de desarrollo específicos.

Mediante el estudio de la perspectiva hirschmaniana centrada en la acción humana y en las complejidades intrínsecas al proceso de toma de decisiones a nivel individual y social en la trilogía del desarrollo se evidenciarán algunos aspectos distintivos de toda la obra del autor. En primer lugar, la necesidad de ampliar el vocabulario técnico de las ciencias sociales para captar diversos tipos de comportamientos humanos que implican racionalidades ocultas para el tipo de enfoque habitual de las disciplinas de la época. En segundo lugar, y en correlación con lo anterior, el traspaso de fronteras disciplinarias continuamente propuesto por Hirschman, y que va desde la economía a las demás ciencias sociales, dialogando, a su vez, con la psicología y la filosofía. Y en este punto, precisamente, creemos que se encuentra uno de los elementos fundamentales de la actualidad de la obra hirschmaniana. En efecto, el enfoque centrado en la acción humana, las tensiones entre las imágenes de cambio, las expectativas y los procesos decisionales de los actores involucrados implican una seria crítica a la teoría económica convencional sobre la elección racional de los agentes. En tal sentido, podría decirse que los constantes intentos de Hirschman, iniciados hace al menos sesenta años y diseminados en toda su obra, por "complicar la economía" (Hirschman 2013) sacando a la luz racionalidades ocultas, consecuencias no deseadas y consecuencias deseadas, pero no alcanzadas de las acciones humanas en los procesos de cambio social, encuentran -al menos en parte- paralelo en los desarrollos más recientes de la Behavioral Economics ${ }^{3}$.

Sobre las posibles relaciones entre el pensamiento hirschmaniano y los científicos conductuales, pueden verse las posturas contrapuestas de Sunstein (2015) y Flyvbjerg (2016). Por otra parte, sobre el sentido en que Hirschman puede ser considerado un economista conductual y, al mismo tiempo, sus diferencias fundamentales respecto de la corriente actual de la Behavioral Economics, véase Hargreaves Heap (2016). 
El estudio de los "sesgos" y las "heurísticas" de decisión por parte de Kahneman y Tversky (Kahneman 2012), así como también las investigaciones en torno a la "fuerza de voluntad" y a la "preferencia por lo justo" de Thaler, tienden a poner en evidencia aspectos psico-sociales vinculados con la toma de decisiones de los agentes económicos que para la teoría económica convencional -según el propio Thaler-suelen tratarse como "factores supuestamente irrelevantes" $(2017,35)$. Sin embargo, tales factores son fundamentales para comprender de qué manera los humanos "de carne y hueso" (y no el Homo Economicus, o los Econs, como los llama) se comportan y, en última instancia, también para elaborar políticas públicas más efectivas.

Al avanzar en la comprensión del proceso de toma de decisiones de los agentes y consolidarse como una de las ramas más promisorias de la economía contemporánea, podría decirse que la economía conductual ha reabierto el debate sobre la consideración de los agentes económicos en cuanto decisores reales. En este contexto, creemos que la lectura integral de la obra de Hirschman puede ser revalorizada y servir como inspiración para el abordaje de problemáticas actuales tanto para la economía como para el resto de las ciencias sociales desde un enfoque metodológico diverso, tanto de la economía convencional como de la conductual, aunque con múltiples posibilidades de complementariedad ${ }^{4}$.

Para dar cuenta de lo anterior, el trabajo procede de la siguiente manera. Después de una breve - pero necesaria- referencia biográfica sobre el autor, se centrará en The Strategy of Economic Development (1958), texto que surge como una primera condensación intelectual que amalgama un complejo y variado cúmulo de vivencias nacionales entre Europa y Norteamérica, una ecléctica formación académica, un particular compromiso prácticoprofesional, y las experiencias del autor a partir de su estancia en Colombia. En este caso, el foco se pondrá en un aspecto que el propio Hirschman, en la segunda edición de la obra, advirtió como poco considerado por la crítica, aunque de gran importancia, i.e., el estudio de las motivaciones para el desarrollo; cuestión que, según aquí se entiende, resulta fundamental para entender la singularidad del enfoque hirschmaniano acerca del desarrollo que, por una parte, produce sus propios aportes para la comprensión del fenómeno, $\mathrm{y}$, por otra, lo conducirá a una discusión constante con la economía convencional y a traspasar las fronteras disciplinares enriqueciendo, al mismo tiempo, las categorías económicas.

Pasando por una breve revisión de las principales críticas de Hirschman a la doctrina del crecimiento equilibrado en la Estrategia, el trabajo continuará identificando la expansión del enfoque del cambio centrado en la acción en Journeys toward Progress: Studies of Economic Policy-Making in Latin America (1963) y en Development Projects Observed (1967), los otros dos componentes de la trilogía. Se concluirá con una síntesis acerca del camino seguido por las ideas hirschmanianas a partir de la Estrategia, señalando su significación de acuerdo con el hilo conductor argumentativo del trabajo, y con unos comentarios finales sobre la relación entre el análisis aquí presentado y el resto de la extensa obra del autor.

\section{Albert O. Hirschman, pionero del desarrollo}

Suele reconocerse que para comprender mejor la obra intelectual de un pensador importa conocer algunos detalles de su trayectoria y experiencia vital. En el caso de Hirschman, bien podría decirse que esta afirmación es particularmente valedera ${ }^{5}$. En su Alemania natal participó siendo muy joven de movimientos antinazis, teniendo que emigrar tempranamente. Con dieciocho años se traslada a $\mathrm{Pa}-$ rís donde comienza sus estudios de economía, para luego continuarlos en la London School of Economics. Como brigadista del ejército republicano lucha en la Guerra Civil Española. En Trieste, Italia, se doctora en Economía y milita en el movimiento antifascista, recibiendo la influencia intelectual de su cuñado Eugenio Colorni. Tras retornar a Francia, se une al ejército $\mathrm{y}$, cambiando su identidad, ayuda a emigrar hacia EE.UU. a refugiados franceses, hasta convertirse en uno de ellos.

\footnotetext{
Otras miradas acerca de la actualidad del pensamiento hirschmaniano pueden encontrarse en Hurtado (2014), Ginzburg (2016), Meldolesi (2017) y, en general, los artículos reunidos por Meldolesi y Stame (2017)

5 Sobre la vida y pensamiento del autor, además de la entrevista de carácter autobiográfico (Hirschman, 1999), deben considerarse como fundamentales los trabajos de Meldolesi (1997) y Adelman (2013a). Además, pueden consultarse los textos de De Haan (2016: 308-349), Blanco (2013), Adelman (2013b), Feinstein (2006) y Furió-Blasco (1998, 7-100).
} 
Ya en Norteamérica, trabaja en la Junta de la Reserva Federal, como especialista en economía europea, en el Plan Marshall desde el 46 hasta el 52, año en el que se traslada a Colombia como asesor del Consejo Nacional de Planeación, organizado por el Banco Mundial, al que luego renuncia para convertirse en consultor privado.

En 1956 retorna a EE.UU. como profesor visitante de la Universidad de Yale y en el 58 publica La estrategia del desarrollo económico, a partir de lo cual permanecerá en el mundo académico (Columbia, Harvard y Princeton) pero sin perder contacto con las realidades de los países subdesarrollados de centro y Sudamérica (Hirschman 1986b).

Bien podría decirse en base a este mínimo esbozo del trayecto vital de Hirschman que vivió varias vidas en una, y que la singularidad de su pensamiento está signada por la multiplicidad de realidades que trató de comprender y transformar. No obstante, si quisiéramos identificar una constante en su derrotero teóricopráctico, un recuerdo de su juventud será revelador:

... descubrí, con gran sorpresa e incomodidad, que mi padre no tenía lo que en aquel entonces yo consideraba una necesidad básica para cualquier persona: ¡una Weltanschauung! Probablemente la historia posterior de mi vida y de mi pensamiento puede ser escrita en los términos del descubrimiento progresivo, por mi parte, de todo aquello en lo que mi padre tenía razón. (Hirschman, Coimbra, marzo de 1993, citado en Medolesi 1997, 7)

En efecto, en gran medida el carácter, la profundidad y riqueza del pensamiento hirschmaniano se comprende en su confrontación ante pretensiosas concepciones totalizantes y holísticas, desafiando la tendencia reduccionista de la razón al revelar la inmensa complejidad de la realidad desde lo particular e inmediato (Adelman 2013, 12-13).

Ahora bien, este talante crítico y desafiante ante las ortodoxias tomó un sentido particular a partir del contacto del economista con las realidades latinoamericanas y su experiencia vital en Colombia. En efecto, Hirschman fue enviado desde el Banco Mundial como un experto para la elaboración de un plan integral de desarrollo del país, en un contexto político internacional en el que el avance del socialismo hacia Latinoamérica presentaba una amenaza real para Estados Unidos. Embarcarse en esa tarea llevó a Hirschman a recorrer buena parte del territorio colombiano, conociendo de primera mano las vicisitudes, pero también las posibilidades, socioeconómicas a través de las distintas y diversas realidades dentro del mismo país. A partir de este conocimiento directo Hirschman elabora un diagnóstico de la economía colombiana ${ }^{6}$, de cara a un proceso de intervención supervisado por el organismo internacional que chocará con las concepciones y propuestas de otros expertos dentro del mismo programa, pero, más en general, configurará la base de la crítica hirschmaniana hacia la economía del desarrollo de la época ${ }^{7}$. De aquí que este período en la vida de Hirschman sea clave no sólo para entender los orígenes de su trilogía del desarrollo, que lo convierte en uno de los "pioneros del desarrollo" (Meier y Seers 1986; Bustelo 1998, 115-131), sino también para identificar una de las fuentes de inspiración constante en el proceso de elaboración y re-elaboración de sus ideas a lo largo de toda su obra.

\section{Acción humana y perspectiva de crecimiento en The Strategy of Economic Development}

En el prefacio de la edición de 1961 de la $E s-$ trategia, Hirschman recuerda una conferencia en la que había dicho: "Mirar el crecimiento desequilibrado significa, en otras palabras, mirar hacia la dinámica del proceso de desarrollo en pequeño" (1961, 12; Adelman 2016), e inmediatamente señala -con cierto desencanto- que la atención de los lectores de su obra se centró notablemente en la contraposición entre crecimiento equilibrado/desequilibrado y en el análisis de los eslabonamientos. Sin

$6 \quad$ Al respecto, cabe señalar su artículo, escrito después de dos años viviendo en Colombia, "La economía y la planeación de la inversión. Reflexiones basadas sobre la experiencia en Colombia” (Recogido en Furió-Blasco 1998, 338-359). Véase, además, Caballero Argáez (2008).

Detalles del periodo colombiano de Hirschman pueden encontrarse en Adelman (2013, 295-324), Meldolesi (1997, 57-139; 2017 , 51-88). Para la actuación de Hirschman en la misión del Banco Mundial y su polémica con Lauchlin Currie, resulta imprescindible el trabajo de Alacevich (2010). Para una mirada crítica sobre las interpretaciones de esta cuestión hechas por Alacevich y Adelman puede verse Sandilands (2015). 
embargo, advierte que otros temas tratados en el libro "no son menos importantes", entre los que menciona los del Capítulo I, referidos a la motivación.

Discutiendo con las concepciones acerca del desarrollo que buscan un "primer motor" que de manera automática conduzca a los países subdesarrollados hacia la senda del crecimiento, Hirschman advierte que tales aproximaciones tienden a ser simplistas, por cuanto, una vez identificado y obtenido el factor que haría las veces de primum mobile, el desarrollo se daría de suyo. De aquí surge el planteo diferencial de su Estrategia:

... el desarrollo no depende tanto de saber encontrar las combinaciones óptimas de recursos y factores de producción dados como de conseguir, para propósitos de desarrollo, aquellos recursos y capacidades que se encuentran ocultos, diseminados o mal utilizados. $(1961,17)$

A partir de esta concepción se comprende que la perspectiva hirschmaniana sobre la planificación del desarrollo se centre en la identificación de "presiones", "mecanismos de inducción" y "eslabonamientos" entre diversos proyectos de inversión, cuya ubicación y secuencialidad será clave $(1961,18)$.

El problema del desarrollo, de acuerdo con lo anterior, no radicará tanto en algunas cosas a obtener, sino en lograr un factor de unión entre los recursos disponibles en pos del desarrollo (Hirschman 1961, 19). Este factor de unión, dice Hirschman: "Parece consistir en una 'perspectiva de crecimiento' que comprende el deseo del crecimiento económico y la percepción de la naturaleza esencial del camino que lleva hacia él" (1961, 22). Ahora bien, dicha "perspectiva de crecimiento" sólo se adquiere gradualmente en y a través del mismo proceso de desarrollo, lo que parece llevar a un razonamiento viciado por circularidad. No obstante, Hirschman aclara que su análisis conduce a una jerarquización entre círculos argumentales: "por sí solo coloca a las dificultades de desarrollo en el lugar donde empiezan y al que pertenecen todas las dificultades de la acción humana: en la mente" $(1961,22)$. De esta manera, el autor invita a pensar el desarrollo a partir de las tensiones entre tres elementos clave: las distintas "imágenes del cambio" de las sociedades, la formación y condicionalidades de las expectativas del cambio, y el proceso concreto de toma de decisiones.
La clasificación hirschmaniana entre dos tipos de "imágenes del cambio" para las sociedades obliga a pensar la elaboración y ejecución de proyectos de desarrollo, teniendo en cuenta el significado que las propias sociedades dan al proceso de cambio. En este sentido, conforma un elemento fundamental de la "perspectiva de crecimiento" antes señalada.

Hirschman distingue entre la imagen de cambio enfocada al grupo (group-focused), y aquella enfocada al individuo (ego-focused). En el primer caso, el cambio se entiende como progresivo sólo si los individuos mantienen sus posiciones relativas y el crecimiento se manifiesta en el conjunto de la sociedad como un todo, de manera tal que la actuación individual diferenciadora es desalentada. Hirschman sostiene que este tipo de imagen puede efectivamente favorecer el desarrollo, aunque bajo ciertas condiciones, puesto que "debe tenerse mucho cuidado para no violar la única 'imagen' de cambio aceptable. La creencia o la sospecha, por muy equivocada que sea, de que un proyecto conducirá al enriquecimiento individual y no a beneficios colectivos, bien puede significar su fracaso" $(1961,24)$. Esto explica que, bajo dicha imagen de cambio, la realización de ciertos proyectos de desarrollo sea difícil de lograr. Pero mucho más difícil aún, afirma Hirschman, es la aceptación de programas a gran escala que impliquen transformaciones estructurales complejas, ya que, por su propia naturaleza, este tipo de programas generan desequilibrios socioeconómicos notables.

La categoría de "imagen de cambio enfocada al grupo" aplicada a los países subdesarrollados de la época permite a Hirschman interpretar, por una parte, el atractivo que representaba el comunismo, en cuanto sistema que se percibía -allende esto fuera realmente asícomo apto para producir tanto un cambio económico progresivo como su orientación hacia el todo de la sociedad. Por otra parte, ayudaba a entender la acción de los gobiernos en su incapacidad para establecer y sostener prioridades de inversión en programas de desarrollo.

Según la imagen enfocada al individuo, en contraposición, las posibilidades de progreso son referidas por el individuo exclusivamente a sí mismo, sin remitirse a lo que ocurra con la sociedad. Hirschman reconoce que, en principio, parecería que esta última imagen fuese más acorde con la idea de la economía convencional acerca de la relación 
entre "búsqueda del mejoramiento de la propia condición" y crecimiento económico. Sin embargo, afirma:

Existen varias razones para pensar que la imagen del cambio enfocada al individuo es enemiga del desarrollo. En primer lugar, el éxito se concibe no como resultado de la aplicación sistemática del esfuerzo y la energía creadora, combinada posiblemente con "un poquito de suerte", sino debido ya sea a una suerte tremenda o a engañar a los demás con ardides bien planeados. $(1961,27)$

Asimismo, recurriendo a la idea del empresario schumpeteriano, afirma que economistas e historiadores "han considerado que el empresario innovador es, principalmente, un egocentrista" $(1961,28)$, y se han olvidado de otra característica "necesaria prácticamente" de dicha figura: "la habilidad para conseguir un acuerdo entre todas las partes interesadas" $(1961,28)$. Esta habilidad para lograr la cooperación es la que proyecta el empresario anteponiendo la imagen de cambio enfocada al grupo sobre la enfocada al individuo, de modo tal que la ausencia de dicha habilidad en los países subdesarrollados puede comprenderse, precisa y paradójicamente, por el predominio de esta última imagen de cambio:

El componente de "relaciones humanas" del espíritu de empresa, el arte de llegar a un acuerdo y de "reclutar" cooperación, seguirán siendo un cuello de botella crítico dentro de toda acción constructiva que pueda realizarse en el desarrollo económico, hasta que la experiencia logre modificar la imagen del cambio exclusivamente enfocado al individuo y llegue a una que reconozca la posibilidad de beneficios mutuos y de un crecimiento general en toda la economía. Una modificación de este tipo se llevará a cabo mediante una experiencia práctica y directa con el desarrollo a medida que éste se va desenvolviendo. Pero esto tiene que hacerse poco a poco, como cualquier corrección de una idea muy arraigada acerca de la naturaleza del mundo en que vivimos. $(1961,30)$

Análogamente, Hirschman sostiene que la tensión entre la aparición o fortalecimiento de incentivos por el mejoramiento económico y unas expectativas exageradas sobre las ganancias de ciertas actividades "novedosas" pueden generar un obstáculo antes que un ali- ciente al crecimiento, dado que, en el afán por "hacerse ricos con rapidez", muchos agentes pueden dejar de lado empresas más relevantes desde el punto de vista del desarrollo (1961, 31). Esta percepción exagerada sobre las oportunidades en muchos casos lleva a rechazar proyectos suficientemente fundamentados por otros que posibilitarían una tasa de retorno mucho más elevada, fenómeno que denomina "preferencia personalizada por la liquidez" (1961, 32).

Mediante su diagnóstico de las oportunidades y obstáculos del cambio -y, por tanto, del crecimiento económico-, Hirschman insiste en la complejidad del fenómeno y en la conveniencia de ubicar en el centro de la preocupación, más que la cuestión de la cantidad de factores, el proceso de toma de decisiones:

las decisiones de desarrollo no se ven frenadas por obstáculos y escasez físicos, sino por las imperfecciones del proceso de tomar decisiones. Así, la teoría y la política del desarrollo se encuentran ante la tarea de examinar cuáles son las condiciones en que, a pesar de estas imperfecciones, se pueden tomar decisiones de desarrollo a través de técnicas que aceleren el paso de mecanismos de inducción. $(1961,37)$

Ahora bien, poner a las decisiones en el centro de la cuestión del desarrollo para Hirschman significa reconocer, en última instancia, que "el problema fundamental consiste en generar y vigorizar la acción humana en cierta dirección", a lo que agrega: "Nuestro enfoque nos lleva a dudar de la existencia de una energía acorralada por obstáculos villanos. Más bien considera que los obstáculos son reflejo de movimientos contradictorios y de la confusión resultante en la voluntad" $(1961,36)$. Precisamente, es este recalar hirschmaniano del problema del desarrollo en la dinámica de la voluntad y de la acción humana lo que aporta una densidad, amplitud y novedad a su investigación de singular carácter para la economía. A este propósito, destaquemos dos puntos en especial.

En primer lugar, reconocer las tensiones y contradicciones intrínsecas al dominio de la acción humana, "corporeiza" -tanto en el sentido de "dar cuerpo", como en el de "se produce en un cuerpo"- la noción de decisión. Noción que la economía convencional suele identificar con el sentido más formalizado, abstracto y determinístico de elección, esto 
es, una discriminación lógicamente consistente entre alternativas perfectamente conocidas y claramente ponderables en un contexto estático. Situación en la cual, como diría Shackle, en rigor no hay lugar para el componente creativo que toda verdadera elección introduce en la historia (Shackle 1976, 45; Hirschman 1986c, 90).

En segundo lugar, la indagación acerca de la acción humana obliga a identificar diversas lógicas en los procesos concretos de toma de decisión en los que se ven imbricadas las aristas económicas, políticas, sociales, culturales, morales, psicológicas, etc. de las realidades humanas, tanto a nivel individual como colectivo, a nivel local, regional y nacional. Así, a diferencia del paradigma que aboga por explicar toda elección desde una lógica económica, la propuesta hirschmaniana tiende a expandir la economía en una confluencia hacia una ciencia social amplia mediante el diálogo interdisciplinario con otros saberes ${ }^{8}$.

Los puntos anteriores ayudan a comprender más acabadamente el alcance de la crítica hacia la economía del desarrollo contemporánea a la Estrategia, particularmente identificada por Hirschman con la doctrina del crecimiento equilibrado. Teniendo esto en cuenta, a continuación se presentan algunos elementos esenciales de dicha crítica, no con la idea de realizar un análisis pormenorizado de la crítica en sí, sino más bien con la intención de mostrar el sentido seguido por la reflexión hirschmaniana centrada en la acción humana y en la comprensión del proceso de cambio. Esto permitirá, posteriormente, ubicar las ideas vertidas en los otros dos textos que conforman la "trilogía del desarrollo" en una línea argumentativa que avanza y se expande desde la economía del desarrollo hasta el estudio de los procesos de toma de decisiones político-económicas y de evaluación y ejecución de proyectos de desarrollo, manteniendo la novedad de un enfoque que pone en tensión el modo convencional de aproximación intelectual -tanto de la economía, en particular, como de la ciencia social, en general- hacia los problemas vinculados con el cambio social, especialmente referidos a las experiencias de los países subdesarrollados.

\subsection{Críticas a la doctrina del crecimiento equilibrado}

El capítulo III de la Estrategia comienza con una reflexión sobre el escaso desarrollo de la teoría del desarrollo -valga el juego de palabras-. Se reconoce que, en comparación con otras áreas de la economía, como las teorías estáticas de los equilibrios (parcial y general), sobre el problema del desarrollo, los economistas "no han sido capaces de construir, y mucho menos de ponerse de acuerdo, sobre una sola cadena ininterrumpida de causas y efectos que explique claramente la transición del 'subdesarrollo' al desarrollo" (1961, 58). Pero una de las cuestiones interesantes que aparece ya en los primeros párrafos de este apartado es el matiz introducido por Hirschman al plantear el problema desde el paso de la estática a la dinámica, por un lado, $\mathrm{y}$, por otro lado, el estado aún menos explorado del enfoque particular que requiere la realidad de los países subdesarrollados. Esto resulta importante puesto que, advierte el autor, la elaboración teórica más ligada a estos problemas ha estado vinculada con el "crecimiento equilibrado [balanced growth] y a la determinación de las prioridades de inversión" $(1961,58)^{9}$.

Desde el inicio, Hirschman explicita enfáticamente su total desacuerdo con la doctrina del crecimiento equilibrado -sostenida por autores como Rosenstein-Rodan, Nurkse, Lewis y Scitovsky- tanto en su versión desde el lado de la oferta como desde el lado de la demanda. Considerando esta última perspectiva, afirma Hirschman: "se dice que para que el desarrollo sea posible es necesario empezar, al mismo tiempo, un gran número de nuevas industrias que serán clientes unas de otras a través de las compras de sus obreros, empleados y dueños. Por esta razón, tal teoría ha necesitado anexarse a la del 'gran impulso' [big push]" (1961, 59). A partir de allí expone sus principales críticas.

Primeramente, destaca la insuficiencia de esta teoría precisamente como teoría del desarrollo, es decir, de un proceso de cambio:

Se supone que el desarrollo significa el proceso mediante el cual un tipo de economía se con-

\footnotetext{
Esto quedará particularmente reflejado en la crítica hirschmaniana a las "pretensiones de la monoeconomía" en un ensayo posterior en el que reflexiona sobre el derrotero de la economía del desarrollo (Hirschman 1984, 13 y ss.)

9 Sobre la contraposición entre crecimiento equilibrado y desequilibrado, véase Bustelo (1998, 122-123) y Alacevich (2016).
} 
vierte en algún otro tipo más avanzado. Pero, en la teoría del crecimiento equilibrado, un proceso de este tipo no puede presentarse ya que le es difícil visualizar el rompimiento del "equilibrio del subdesarrollo" en cualquiera de sus puntos. $(1961,59)$

Haciendo caso omiso de las múltiples diferencias entre los diversos sectores y actividades en los países subdesarrollados, la teoría del crecimiento equilibrado, continúa Hirschman, concluye que "debe imponerse una economía industrial moderna, enteramente nueva y completa, sobre el sector tradicional estancado e igualmente completo" $(1961,60)$. No obstante, más bien que "crecimiento", lo que se logra mediante esto es un "patrón perfectamente dualista del desarrollo" (1961. 60). Precisamente, es este carácter dualista, que concibe a las economías tradicionales y a las modernas como "círculos cerrados", desde una perspectiva estática, o de yuxtaposición, el punto más débil de la teoría del crecimiento equilibrado, según Hirschman.

Sintetizando su principal crítica, el autor advierte sobre la inconsistencia fundamental, a nivel teórico, a la vez que sugiere una inquietante derivación práctica:

Ésta es la crítica principal que quiero hacerle a la teoría del crecimiento equilibrado: su aplicación requiere precisamente montos enormes de aquellas capacidades cuya oferta es muy limitada en los países subdesarrollados. Es enteramente inconcebible que una economía de un sólo piso erija un "segundo piso" de este tipo con sus propias fuerzas o aun con una ayuda limitada del exterior; sin una colonización extranjera completa, la tarea parecería irrealizable. [...] En otras palabras, si un país pudiera aplicar la teoría del crecimiento equilibrado no estaría subdesarrollado. (1961, 61; cursiva añadida)

La idea de una estrategia para el desarrollo basada en decisiones de inversión que apro- vecharan diversas tensiones y desequilibrios entre diferentes sectores, regiones y actores en los países subdesarrollados, poner en juego distintos mecanismos de inducción y las secuencialidades propias de los eslabonamientos, en fin, pensar el desarrollo en pequeño, contrastaba fuertemente con la grandilocuente concepción de "planificación integral", heredera de los "remedios keynesianos" (de la síntesis neoclásica) para países desarrollados en períodos de crisis (Hirschman 1984, 20) ${ }^{10}$. Esta crítica a la planificación integral -una concepción de planificación consistente desde la perspectiva hirschmaniana con la noción de crecimiento equilibrado-, sumada a su énfasis en las decisiones, puede llevar a pensar que el autor era totalmente reacio a la planificación sin más y, en general, a la intervención estatal en economía, lo que sería una inferencia errónea ${ }^{11}$.

Destacar los problemas para "generar y vigorizar la acción humana en cierta dirección" significa, ante todo, reconocer que la complejidad de la toma de decisiones se encuentra tanto en la economía como en la política, de manera tal que la idea de "reemplazar mercado por Estado" no es para Hirschman una opción real, sino más bien un reduccionismo teórico con graves consecuencias prácticas ${ }^{12}$.

Lo anterior apunta a otra de las cuestiones constantes en la Estrategia -y en el conjunto de la obra hirschmaniana-: el feedback permanente entre la captación de complejidades a partir de la observación y vivencia de las realidades a comprender y enfrentar, y la crítica hacia las estructuras teóricas preconcebidas con las que el científico o el experto intenta entender y modificar dichas realidades.

En el capítulo II de la Estrategia, Hirschman había advertido sobre la tentación que representaba para la economía del desarrollo apropiarse de los avances de la economía del crecimiento, particularmente representados en la época por los estudios de Harrod y Domar, dado el nivel de abstracción y las condicionalidades dentro de las cuales estos modelos pueden ser de utilidad,

10 Sobre esto puede verse el trabajo de Boianovsky (2015). Asimismo, en un artículo posterior, Hirschman reconoce las semejanzas, pero más aún las diferencias, de su enfoque de los eslabonamientos con respecto al pensamiento marxista, caracterizando su posición como "micromarxista" (Hirschman 1984, 118 ss.). Por otra parte, sobre las relaciones entre los planteos hirschmanianos y los de autores vinculados con la CEPAL, pueden consultarse los trabajos de Ocampo (2008), Santiso (2000) y del propio Hirschman $(1973,88-123$ y $260-296)$.

11 Tal como parece sugerir Sandilands $(2015,215)$ que es la interpretación de Easterly, acercando la posición de Hirschman a la de Hayek.

12 A propósito, el propio Hirschman se encargó de diferenciar su posición sobre la identificación de racionalidades ocultas y consecuencias no previstas de las acciones respecto del reconocido anti-intervencionismo de Hayek (Hirschman 1999, 83-84). 
que, por supuesto, difieren en gran medida de las situaciones de los países subdesarrollados:

Por lo tanto, mientras más útiles sean [tales teorías] en condiciones dadas, menos útiles serán, probablemente, en condiciones por entero diferentes. Si a pesar de todo tratamos de "aplicarlas" pueden convertirse en largas desviaciones en lugar de atajos. Como nos hemos acostumbrado a ver la realidad a través de ciertos lentes teóricos, probablemente pasará mucho tiempo antes de que la podamos captar como realmente es. (1961, 39; cursiva añadida)

De manera tal que las reflexiones acerca del desarrollo en torno a la acción humana obligan a indagar más profundamente en los procesos decisionales de los agentes $\mathrm{y}$, al mismo tiempo, a cuestionar la elaboración teórica del científico, en cuanto producto de la propia acción humana y, por tanto, sujeta a similares posibilidades, sesgos y limitaciones (Hirschman 1986a, 99, 102; 1984, 34-39).

\section{Expansión del enfoque hirschmaniano del cambio social a las decisiones políticas}

En Journeys toward Progress: Studies of Economic Policy-Making in Latin America ${ }^{13}$, publicado en 1963, Hirschman intentaba conocer algo de "la capacidad de las autoridades públicas de América Latina para resolver problemas, de las condiciones más propicias para provocar y fomentar esa capacidad y de las formas típicas de enjuiciar y actuar que se irían configurando" $(1964,3)$. Dicho de otra manera, trataba de adentrarse en la comprensión de la comprensión de una serie de problemas vinculados con el desarrollo por parte de las autoridades de los países latinoamericanos -la inflación en Chile, la tenencia de la tierra en Colombia y el desequilibrio regional en Brasil- y las peculiaridades del proceso de toma de decisiones consecuentes ${ }^{14}$. En términos de la Estrategia, podría decirse que intentaba captar la imagen que de las problemáticas se hacían las autoridades para identificar ciertas características de las lógicas -aparentemente ilógicas- decisionales de las autoridades públicas latinoamericanas (Hirschman 1964, 4).

Consciente tanto de las dificultades que su empresa acarrearía como de la relevancia de tal estudio, Hirschman por un lado reconoce ya en la "Nota del autor" que el libro trasciende las fronteras de la economía para ubicarse en una intersección con otras ciencias sociales, y, por otro, vuelve a criticar -como lo había hecho en la Estrategia- la escasa atención que se ha dado al estudio del proceso de toma de decisiones dentro de las ciencias sociales en general $(1964,7)$, de las teorías del desarrollo en particular $(1964,5)$ e incluso de los inconvenientes en su tratamiento dentro de la propia teoría de la empresa en versión schumpeteriana $(1964,6)$.

Hirschman apunta ahora especialmente a la incapacidad de las ciencias sociales para dar cuenta de la complejidad del proceso de cambio como tal, es decir, para captar la dinámica implícita en el proceso decisional concreto:

El deseo de prescindir de la hipótesis de un impulso inicial y la impresión de que las mareas y contramareas del proceso de adopción de decisiones desempeña un papel importante en todas las fases del desarrollo me indujo antes a investigar varios mecanismos (desequilibrios, eslabonamientos, etc.) que causan esas mareas, es decir, que extraen del proceso de desarrollo cantidades extraordinarias de decisiones en el plano de la empresa y de la administración. Este leitmotiv de mi obra anterior no se limita en modo alguno a esa función en lo privado. Afirmé que fuerzas ajenas al mercado $[\ldots]$ no son por fuerza o intrínsecamente menos automáticas que la reacción de un empresario privado ante un aumento del precio de sus productos. El deseo de documentar esta afirmación ha determinado el tema principal de esta obra; a saber: investigar el comportamiento de las autoridades públicas en posición de decidir ante situaciones que exigen la solución de un problema. $(1964,6)$

De esta manera, lo que en la Estrategia había sido un llamado de atención para evitar re-

13 La traducción castellana que aquí se utiliza lleva como título: Estudios sobre política económica en América Latina (En ruta hacia el progreso) (1964).

14 Cabe señalar aquí que en 1961 se publica una compilación de trabajos, bajo la edición de Hirschman, con el título de Latin American Issues. Essays and Comments. En el artículo de Hirschman, "Ideologies of Economic Development in Latin America", aparece la idea de la necesidad de los asesores extranjeros de comprender la manera en que los latinoamericanos comprenden su propia realidad: "But frequently our advice will be futile unless we have also gained an understanding of the understanding Latin Americans have of their own reality." $(1961 b, 4)$. 
caer en una explicación de tipo primum mobile para el desarrollo, ahora se generaliza al resto de las ciencias sociales apuntando a las habituales respuestas al problema de la transición del mal al buen gobierno. La crítica se dirige, por tanto, a la dicotomía buen/mal gobierno en tanto asociada de modo determinista a una estructura socio-institucional dada (Hirschman, 1964, 7).

Tratando de trascender la escisión entre estructura y decisiones, es decir, reforzando la idea de interdependencia e influencia bidireccional, la propuesta de Hirschman aboga por dilucidar la significación y resultados de las decisiones de las autoridades públicas de los países subdesarrollados tanto a partir de los condicionamientos que enfrentan desde las estructuras en las que se llevan a cabo, como desde las propias posibilidades para modificar dichas estructuras. De esta forma, sugiere, se tendría una visión más acertada de las implicancias y consecuencias de los procesos de cambio según las particularidades que adopta en los países latinoamericanos, lo que, a su vez, ayuda a no caer en un pesimismo determinista ni en un optimismo voluntarista a la hora de abordar el problema de las capacidades reales de estos países para progresar política, social y económicamente $(1964,8)^{15}$.

En este contexto, Hirschman expone enfáticamente su intención de evitar una salida fácil, simplista o reduccionista de la 'paradoja del cambio', i.e., “¿Cómo puede tornarse el mal gobierno en bueno, la reacción en reforma, la parálisis en progreso?" A lo que responde:

Como ya lo intenté en mi obra anterior sobre desarrollo económico, mi propósito es responder a estas preguntas sin caer en el tentador recurso -o juego de manos- de descubrir un requisito previo, ya sea la presencia de recursos naturales, una tasa de formación de capital, una minoría selecta, una ideología o una personalidad estructurada de cierta manera que, según algunos, se debe dar antes que se pueda efectuar la transformación. Antes bien: trataré de demostrar la forma en que una sociedad puede ponerse en marcha tal como es, a pesar de cómo es $\mathrm{y}$ precisamente por lo que es. $(1964,9)$

Junto a esta exposición de su enfoque general, Hirschman especifica la metodología que seguirán sus indagaciones mediante una aproximación al análisis del proceso de decisiones políticas desde dos flancos: por un lado, los intentos de neutralizar-superar obstáculos reconocidos y, por otro, identificar los aspectos progresivos ocultos que pudieran presentar ciertas condiciones y actitudes generalmente valoradas como reaccionarias. Ahora bien, como el mismo autor se encarga de aclarar, la adopción de tal metodología no obedece ni a un sesgo hacia el gradualismo político ni a una particular inclinación por lo paradójico, sino que se impone por su propia concepción de la dinámica de los procesos decisionales intrínseca al cambio social:

[S]i se quiere prescindir del concepto del requisito previo único y universalmente necesario, no se podrá comprender ni concebir el cambio, a menos que se demuestre que algunos ingredientes del viejo orden son ambivalentes y poseen en potencia elementos que pueden fomentar el progreso y el crecimiento. De ahí mi gran atención por el bien, que por mal viene, y mi interés de coleccionista por constelaciones que permitan sacar fuerza de flaqueza, no obedezca a una infatuación con la paradoja; antes bien, vienen determinados por la esencia del proceso de cambio tal como lo entiendo. $(1964,10)$

Ahora bien, la aclaración que Hirschman realiza con relación a la metodología seguida en el texto de 1963, y que, como puede notarse, corresponde a una extensión de aquella seguida en la Estrategia, no pretende quedarse a un nivel estrictamente teórico-analítico, sino que tiene como finalidad última contribuir a una mejora concreta en el proceso de toma de decisiones por parte de los actores responsables, i.e. la aproximación científica social hirschmaniana se presenta como ciencia práctica. En este sentido, afirma:

Mantengo que esta idea permite comprender mejor el proceso de desarrollo. Y lo que es más importante, si las personas que están llamadas a tomar decisiones de política pudieran llegar a reconocer esta idea, es probable que llegaran a percibir con mayor claridad los varios caminos que pueden seguir y realzar su capacidad y astucia. $(1964,10)$

15 Rodrik destaca la concepción "posibilista" de Hirschman contra una perspectiva determinista en ciencias sociales (2016, 215). Acerca del posibilismo hirschmaniano y su metodología, véanse los trabajos de Meldolesi (1997, 15-54), Lepenies (2008) y Valencia Agudelo (2013). 
La comprensión hirschmaniana de la dinámica del cambio social le permite obtener una serie de generalizaciones a partir de su estudio de casos, como por ejemplo la función de la ideología en la selección de problemas a resolver por parte de las autoridades y el recurso a la sustitución de un problema por otro, la desproporción entre la motivación para resolver un problema y la comprensión del mismo, la precipitación hacia la pseudo-comprensión del problema -lo que con Flaubert llama "la rage de vouloir conclure"-, y una de sus expresiones más conocidas, el complejo de fracaso o "fracasomanía"; cuestiones cuyo análisis exhaustivo exceden los límites de este trabajo, pero en las que importa identificar el espíritu del enfoque hirschmaniano de la acción humana ${ }^{16}$.

Cada una de las observaciones que Hirschman generaliza a partir del estudio de secuencias concretas de problemáticas vinculadas al desarrollo en países latinoamericanos son esfuerzos para captar los desequilibrios y desproporciones propias de las capacidades cognitivas del ser humano, de las contradicciones y confusiones de la voluntad, y de las múltiples y complejas relaciones entre ambas dimensiones. Este enfoque, por tanto, obliga a cuestionar las categorizaciones duales que las ciencias sociales suelen utilizar, como la mencionada entre buen/mal gobierno en política o el comportamiento racional/irracional en economía. Igualmente, permite cuestionar la oposición entre revolución violenta y reforma pacífica. Más allá de la importancia que el tratamiento de este tema tenía en el contexto histórico en que aparecen los Estudios sobre política económica en América Latina, lo que aquí interesa destacar es la simplificación que tal oposición implica y, por tanto, las complicaciones que esto conlleva para alcanzar el progreso.

En principio Hirschman aclara que, en rigor, no puede pensarse una dinámica de cambio progresivo sin modificar la situación percibida como "de privilegio" de al menos un grupo social; lo que generará, por ende, algún tipo de resistencia:

En verdad, todo "progreso", por poco antagónico que se pretenda, casi siempre ha de perjudicar, por lo menos al principio, la posición absoluta o relativa de algún grupo social. Los antropólogos nos han enseñado que todos los aspectos de un status quo, por indeseables que sean, siempre tienen quien los defienda o se aproveche de ellos, por cuyo motivo se opondrá a toda tentativa de mejorar. $(1964,284)$

No obstante, al mismo tiempo señala que lo anterior no significa defender el ideal revolucionario, dado que la propia noción de revolución suele presentarse como producto de la incapacidad humana para comprender y lidiar con el proceso de cambio progresivo como tal:

La idea de que la revolución es el requisito previo de todo progreso cuenta con un sólido apoyo en la limitadísima facultad humana de imaginar visualmente el cambio y en el hecho de que exige muy poco de esa facultad. Lo único que nos pide el revolucionario es que imaginemos el derrocamiento del antiguo régimen en una conmoción total que dará a luz un nuevo orden. Así concebida, la revolución es en esencia un breve pero cataclísmico interludio entre dos sociedades estáticas. $(1964,287)$

Análogamente a la crítica que Hirschman hacía en la Estrategia a la ortodoxia del crecimiento equilibrado, se despliega ahora el cuestionamiento a la ortodoxia de la heterodoxia política, es decir, a la concepción que sólo concibe el cambio desde la revolución. El problema de base es el mismo: la inadecuación entre dos visiones opuestas -pero igualmente reduccionistas sobre el proceso de cambio social- ante la complejidad de la dinámica concreta. Y como se ha dicho, este problema no se reduce a una cuestión meramente teórica, sino que permite entender en la práctica la situación de los países subdesarrollados. En palabras del propio Hirschman: "A juicio del autor, este contraste entre la realidad y la imagen de la realidad es un fenómeno que prevalece hoy en gran parte de la América latina" $(1964,289)$.

\section{Ampliación de criterios de evaluación en proyectos de desarrollo}

En el último de los textos correspondientes a la "trilogía del desarrollo", Development Projects Observed (1967) ${ }^{17}$, el enfoque hirschma- 
niano anclado en la acción humana se dedica al estudio de once experiencias concretas de proyectos de desarrollo en diferentes sectores de la economía de diversos países subdesarrollados, con el propósito de identificar lo que denomina ciertas "características estructurales" de tales proyectos, entre las que se cuentan las capacidades económico-tecnológicas como así también sus propiedades organizativo-administrativas $(1969,4)$.

La identificación de esas características estructurales, según Hirschman, es fundamental por dos motivos. En primer término, porque permitirá ampliar la comprensión tanto del hecho como de las posibilidades del éxito o fracaso de los proyectos. En segundo término, porque sugiere que el desarrollo de un país en cierta forma depende del tipo de proyectos que se acepten o promocionen. Dice el autor:

Este enfoque pone de relieve la importancia del desarrollo de lo que un país hace y de lo que llega a ser como resultado de lo que hace, y de este modo refuta la primacía de lo que es, o sea, de la dotación de recursos naturales, valores, instituciones, estructura social y política, etc. determinada por su geografía y su historia. $(1969,4-5)$

El pasaje de una concepción estático-normativa de "las cosas que hay o debe haber" a una concepción dinámico-positiva de "las decisiones que se toman o pueden tomarse", muestra su potencial ahora en el estudio de proyectos específicos, cuya ejecución repercutirá - para bien o para mal- en el proceso de transformación del país en su conjunto. Desde esta perspectiva, así como antes el enfoque hirschmaniano centrado en la acción reclamaba una revisión de la teoría económica del desarrollo equilibrado y de la dicotomía política de buen/mal gobierno, ahora la crítica se dirige a los criterios habitualmente utilizados para el análisis y evaluación de proyectos.

Hirschman entiende que también en el caso de la evaluación de proyectos se ha planteado una dicotomía entre un aspecto técnico-cuantitativo de la evaluación, que consistiría en la determinación de una tasa de rentabilidad, y otro aspecto vinculado con la decisión en sí, aunque vagamente librado al sentimiento o la intuición. Esta dicotomía, por tanto, contribuye a ocultar otras dimensiones intrínsecas al proceso de análisis-ejecución de proyectos en su relación con el desarrollo del país en su conjunto. Así, la crítica de Hirschman no apunta tanto a abandonar los criterios convencionales de evaluación, sino más bien a la necesidad de complementarlos con otras consideraciones relativas al razonamiento práctico: "Más que como una crítica del análisis de la relación costo beneficio y la tasa de utilidad, este libro debe considerarse como un intento de reconquistar siquiera una parte de este vasto campo de la discreción intuitiva para los procesos usuales de la razón raciocinante" $(1969,8)^{18}$.

El estudio de los desequilibrios, las secuencias, las confusiones y contradicciones de las decisiones, se llevan ahora al ámbito de las posibilidades compensatorias que se despliegan en, y a través de, los proyectos de desarrollo. En efecto, en principio Hirschman parece identificar una verdad de perogrullo, i.e., que los proyectos pueden presentar una serie de amenazas que conspiren contra su desenvolvimiento, pero también pueden conllevar un conjunto de decisiones superadoras cuando alguna de tales amenazas se concreta (1969, 11). Ahora bien, aquí interesa destacar tres elementos. En primer lugar, la transición del carácter potencial al actual de las amenazas y de las decisiones superadoras. En segundo lugar, lo "insospechado" tanto de las posibles amenazas como de las decisiones superadoras, dicho de otra manera, su carácter sorpresivo y esquivo al conocimiento. Por último, donde el autor pondrá más énfasis, la posibilidad de una compensación -parcial o total- entre amenazas y decisiones en el devenir del proyecto.

A partir de lo anterior, Hirschman llega a la idea de que la dinámica creativa de las decisiones de los proyectos de desarrollo muestra una doble subestimación compensatoria (1969, 12-13): por un lado, una subestimación de las amenazas potenciales, y por otro, una subestimación a las capacidades de los agentes para tomar decisiones superadoras una vez que ciertas amenazas se concretaron. Sin esta compensación, muchos de los proyectos analizados no se hubieran llevado a cabo, de modo que si hubiera habido un mayor conocimiento sobre las problemáticas futuras, muchas acciones en pro de los emprendimientos de desarrollo no se hubiesen concretado. A los efectos benéficos de esta especie de ocultamiento de 
las amenazas como aliciente a la toma de decisiones, Hirschman le da estatuto de principio con nombre propio, la Mano Encubridora:

En otras palabras, puesto que necesariamente subestimamos nuestra creatividad, resulta deseable subestimar en medida aproximadamente semejante las dificultades de las tareas que enfrentamos, de tal modo que estas dos subestimaciones compensatorias nos envuelvan en empresas que somos capaces de realizar, pero que no nos atreveríamos a emprender de otro modo. El principio es suficientemente importante como para merecer un nombre: puesto que aparentemente nos encontramos en la esfera de la influencia de alguna especie de mano invisible que nos esconde benéficamente las dificultades, propongo que se le denomine la Mano Encubridora. $(1969,13)$

Utilizando la terminología de la Estrategia, podría decirse entonces que la Mano Encubridora funciona mediante una imagen de cambio errónea, o al menos borrosa, que genera expectativas exageradas. Pero, a diferencia de los efectos negativos que Hirschman adscribía a la exageración de expectativas en aquella oportunidad, en este caso señala la posibilidad de su actuación como un mecanismo impulsor para la toma de decisiones creativas en conjunto, lo que -como se dijo- apunta al problema fundamental para el progreso de los países subdesarrollados: "En otras palabras, en los países desarrollados se requiere menos disfraz de las incertidumbres y probables dificultades de una tarea prevista que en los subdesarrollados, que carecen de confianza en la creatividad" $(1969,15)$.

Este énfasis en las tensiones entre expectativas e incertidumbres $(1969,36-37)$, por una parte, e impulso para la toma de decisiones creativas, por otra, que subyacen a la acción de la Mano Encubridora, ayudan a identificar y entender la existencia de ciertos sesgos en la selección de proyectos $(1969,17)$. Estos sesgos se ponen de manifiesto al considerar diversas estrategias que los agentes utilizan de facto, a la hora de proponer determinados proyectos, para generar cierta confianza -es decir, tratando de superar el escenario de incertidumbre señalado-y lograr así su evaluación positiva y posterior ejecución.

Entre este tipo de estrategias, Hirschman menciona las técnicas de la "seudoimitación" y de "programa seudocomprensivo" (1969,
21-22). Mientras que la primera consiste en minimizar la incertidumbre y acelerar el proceso de decisiones asociando el proyecto en cuestión al supuesto éxito que la aplicación de una técnica ha tenido en el extranjero, la segunda apela a un supuesto conocimiento más abarcador de la solución propuesta por conformar un "programa comprensivo". Ambas técnicas funcionan de manera complementaria, dado que la primera sugiere una disminución de las dificultades potenciales, mientras que la segunda intenta persuadir acerca de un incremento en el conocimiento de las posibles amenazas $(1969,25)$. Como se dijo, ninguna de estas situaciones es real, sino que son imaginadas e intencionalmente propuestas para su aceptación -en tal sentido, sesgadas-, pero ayudan a poner en marcha el proceso de toma de decisiones y la actividad emprendedora.

De esta manera, así como para Aristóteles conocer lo bueno no alcanzaba para obrar lo bueno, y se llegaba a ser virtuoso -i.e., a poseer el hábito bueno- practicando concreta y sucesivamente acciones buenas, para Hirschman la falta de conocimiento completo de las situaciones actuales y futuras de los proyectos no impide -vía la Mano Encubridora- su ejecución, y la propia puesta en práctica de los proyectos particulares, con el abultado y complejo entramado de decisiones que presuponen, va generando un hábito en el mismo proceso decisional que permitiría abandonar el círculo vicioso entre el temor a lo desconocido (incertidumbre) y la atrofia de la voluntad (indecisión):

uno de los beneficios indirectos de los proyectos consiste precisamente en el aumento de la disposición de quienes toman las decisiones a enfrentar las dificultades e incertidumbres. La Mano Encubridora constituye esencialmente un mecanismo que permite que el temeroso de los riesgos los acepte y que en el curso del proceso se vuelva menos temeroso. De esta manera, ofrece una forma de escapar de algunos de los más formidables "prerrequisitos" o "precondiciones" del desarrollo; permite que los llamados prerrequisitos surjan después de que ha ocurrido el acontecimiento del cual se suponía que constituían un prerrequisito. $(1969,26)$

Al retomar, en la última parte de la cita anterior, la crítica de la Estrategia a la búsqueda del primum mobile, Hirschman sugiere magistralmente la conexión entre, por un lado, 
su anclaje en el estudio de la acción humana y la preocupación -teórica y práctica- por el desarrollo y, por otro, la expansión que su enfoque exige a la evaluación convencional de proyectos. En efecto, un poco más adelante afirma que "la contribución de las empresas de desarrollo depende no sólo de sus resultados financieros, adecuadamente separados, sino de los importantes efectos laterales que a menudo se reflejan en la secuencia temporal de estos resultados" (1969, 27). Esos efectos laterales (1969, 156-183), en gran medida se vinculan con el fortalecimiento del "espíritu de empresa" $-\mathrm{O}$, continuando con la analogía aristotélica, podríamos llamar "hábito de empresa"-, que la propia secuencia de enfrentar una amenaza y superarla, ayuda a mantener y amplificar, en el mejor de los casos.

Ahora bien, puesto que el enfoque hischmaniano centrado en la acción humana trata de echar luz sobre los mecanismos que inducen el proceso decisional, esto no implica -antes, todo lo contrario- caer en un mecanicismo de la acción. Es por ello que, así como destacó las consecuencias positivas que puede acarrear la Mano Encubridora, Hirschman también llama la atención sobre sus riesgos ${ }^{19}$. Los sesgos sobre los que dicho principio se sustenta no logran un resultado prometedor de manera determinística, sino que pueden llevar al fracaso de los proyectos e incluso al establecimiento de "hábitos malos" por el abuso de las técnicas de seudoimitación y de programa seudocomprensivo. En este sentido, Hirschman deja en claro que la Mano Encubridora es esencialmente un mecanismo de transición a través del cual quienes toman decisiones aprenden a aceptar riesgos, y "mientras más corta sea la transición y más rápido el aprendizaje, será mejor" $(1969,27)$. Recordando a Maquiavelo, podría decirse que el objetivo de la Mano Encubridora en el proceso de desarrollo de los países debería ser disminuir sucesivamente el ámbito de acción de la fortuna incrementando el rango de acción de la virtù. De esta manera, queda claro que el sentido de actuación de la Mano Encubridora corresponde, en última instancia, a la evaluación prudencial del razonamiento práctico $(1969,29$ y 85).

\section{Conclusiones}

En la última parte del capítulo dedicado a la Mano Encubridora en Development Projects Observed, Hirschman vuelve sobre la tensión entre imagen-decisión para la comprensión del proceso de cambio. Tensión que, como se ha tratado de mostrar hasta aquí, se encuentra en la base de la comprensión hirschmaniana del desarrollo en The Strategy of Economic Development, y adquiere su dimensión y significación política en Journeys toward Progress. No obstante, en este caso la cuestión se vislumbra desde el marco más general de la antropología filosófica:

La Mano Encubridora, o, en su ausencia, la exageración de los beneficios, se han considerado útiles como medios para tratar cierta falta de firmeza específica y temporal de ciertas sociedades, es decir, el conocimiento inadecuado del hombre de su propia capacidad para enfrentar dificultades. Un pesimismo mucho más general sobre la naturaleza humana a la cual juzgaba propensa a la debilidad, tendiente a la rutina e inclinada a la decadencia llevó a Georges Sorel a creer que la humanidad necesita "mitos" (imágenes inspiradas de batalla y triunfo) para cualquier movimiento hacia adelante. $(1969,30-31)$

No obstante, Hirschman critica el pesimismo de Sorel, puesto que al poner énfasis en la desproporción entre el hecho cumplido y la imagen previamente formada, este pensador reniega de la posibilidad de una comparación entre ambos. Dicho de otra manera, Sorel prohibía la revaluación de proyectos. Ante esta línea argumental, Hirschman retoma una cita de Kolakowski en la que aparece un razonamiento similar al de la Mano Encubridora y la necesidad de los mitos de Sorel, pero antes de poner el acento en la desproporción entre la imagen y el hecho cumplido, tanto Hirschman como Kolakowski ponen el acento en el efecto motivacional, dinamizador, de la imagen: "los agentes subestiman la fuerza que existe en ellos; por tanto, se considera "imposible" el esfuerzo a realizar hasta que se genera, gracias a la imagen, la energía social necesaria." $(1969,32)^{20}$.

19 Una advertencia similar hará posteriormente en sus "Confesiones" refiriéndose a la Estrategia: "No cabe duda de que se puede ir demasiado lejos en la estrategia del crecimiento desequilibrado, con consecuencias terribles." (Hirschman 1986a, 99).

20 Pero Hirschman va más allá que Kolakowski al identificar no sólo la importancia de la imagen de éxito total o de finalización del cambio para motorizar el proceso decisional, sino también las limitaciones para identificar y valorar los "resultados intermedios o los éxitos parciales" alcanzados en la transición hacia el estado final $(1969,32)$. 
Resulta paradójico que en el texto de la trilogía que, tanto por su objeto como por su finalidad, podría considerarse más puntual y concreto, se encuentre una de las presentaciones más generales y abstractas del enfoque hirschmaniano acerca del cambio social y la dinámica de la acción humana. Pero, siendo el recurso a la paradoja tan caro al propio autor, lo anterior no debe causar extrañeza, sino más bien interpretarse como signo de la relevancia de lo hallado.

Llegados a este punto podría decirse que atravesar los meandros de la trilogía del desarrollo de Hirschman siguiendo el norte de las sucesivas profundizaciones en la elucidación del problema del cambio social mediante las tensiones intrínsecas a la acción humana, ha sido una ardua travesía que, como tal, debe dejar varias enseñanzas. En primer lugar, el haber puesto de manifiesto un principio de unidad para el complejo plexo de términos específicos utilizados por el autor. En efecto, ideas presentes en la Estrategia, tales como "crecimiento desequilibrado", "mecanismos de inducción", "eslabonamientos"; en los Estudios, como las de "precipitación hacia la pseudo-comprensión del problema" o "fracasomanía"; y en el Comportamiento, como "técnicas de seudoimitación y de programa seudocomprensivo" y "Mano Encubridora"; revelan sus múltiples facetas de complementariedad a la luz del enfoque hirschmaniano del cambio y la acción humana, en cuanto se presentan como diversos modos de aproximarse a la comprensión de la transición entre estadios sociales mediante el estudio del proceso concreto de toma de decisiones y, con ello, de los mecanismos cognitivos y volitivos que influyen y se ven influidos en y por el proceso mismo.

En segundo lugar, que la propia preocupación por comprender más acabadamente el cambio, su sentido y consecuencias, lleva a Hirschman a criticar, intentar trascender, o al menos complementar las categorías analíticas de la economía y, en general, de la ciencia social de la época. Como pudo verse en este periplo, tanto el concepto de desarrollo económico, como la dicotomía mal/buen gobierno, y los criterios de evaluación de proyectos, resultaron insuficientes desde la perspectiva teórica del cambio y práctica del progreso centradas en la acción humana a través de la experiencia concreta en países subdesarrollados. Esto conlleva, a su vez, al menos dos cuestiones fundamentales. Por una parte, el constante desafío hirschmaniano a los saberes establecidos y a las fronteras disciplinarias. Por otra, la posibilidad enriquecer a la ciencia social en general, y a la economía en particular (Hirschman 2013), a partir de la reflexión en y sobre experiencias vitales y realidades económicas, sociales y políticas muy diversas a las de los países desarrollados (Hirschman 1984, 21).

Por último, cabría llamar la atención sobre la fecundidad del enfoque hirschmaniano del cambio y la acción humana, en cuanto camino iniciado en la Estrategia. Este aspecto puede abordarse al menos desde dos frentes. Por un lado, considerando los desarrollos del propio autor con posterioridad a la trilogía, $y$, por otro, señalando la actualidad de las ideas hirschmanianas para la economía contemporánea. Avanzar mínimamente sobre cualquiera de estos dos frentes excedería por mucho las pretensiones de estas palabras conclusivas. No obstante, permítasenos finalizar con algunas ideas, a modo de sugerencias para futuras líneas de investigación, en ese sentido.

Con respecto a la producción intelectual de Hirschman subsiguiente a la trilogía, puede decirse que -corsi e ricorsi mediante, recordando a Vico- continúan la senda expansiva del enfoque original al intentar esclarecer la dinámica del cambio social, recurriendo para ello a la observación empírica directa o histórica, creando o resignificando conceptos tendientes a captar facetas ocultas o esquivas de los fenómenos estudiados y cruzando constantemente las fronteras disciplinarias. Así, por ejemplo, en Exit, Voice and Loyalty (1970) tratará de comprender la dinámica de empresas, organizaciones y Estados a partir de la tensión entre su deterioro y las respuestas que ante esa situación las entidades pueden brindar. Este estudio aporta elementos importantes para comprender la acción colectiva que luego serán retomados y ampliados desde una perspectiva diacrónica en Shifting Involvements (1982), en el que tratará de identificar los mecanismos detrás de los ciclos que presentan las sociedades en torno a la vida privada y la participación pública, es decir, a la dinámica social oscilante entre el interés individual y la acción colectiva.

La pregunta por el cambio social, particularmente orientada al entendimiento de las consecuencias políticas del crecimiento económico, llevaron a Hirschman al ámbito de la historia de las ideas. En The Passions and the Interests (1977) trata de comprender el proceso por el cual las actividades, actitudes y modos de vida 
vinculados al lucro pasaron de ser mal vistos -o directamente despreciados- en la historia occidental, a ser ampliamente aceptados y justificados, incluso antes del despliegue del sistema capitalista como tal. Aquí el análisis de las consecuencias no deseadas de ciertas acciones, como así también las consecuencias deseadas, pero no alcanzadas, es decir, la tensión imagendecisión, será clave tanto para criticar otras teorías acerca del surgimiento y expansión del capitalismo, como para -y esto es lo más importante- comprender las posibles consecuencias políticas de ciertos argumentos en favor de un sistema económico. Precisamente en The Rhetoric of Reaction (1991) Hirschman pone en el centro de su análisis a la argumentación política relativa al cambio social, identificando dos grupos de argumentos - cada uno conteniendo tres tesis fundamentales y contrapuestas-, un grupo tendiente a justificar el cambio y el otro dedicado a rechazarlo. De esta manera puede verse que en el último de sus grandes ensayos, Hirschman vuelve sobre otras aristas de una temática que no sólo lo acompaña al menos desde la Estrategia, sino que además moldeó un singular y fecundo estilo de abordaje hacia los problemas elementales de las ciencias sociales.

Por último, a modo de reflexión final acerca de la actualidad del pensamiento de Hirschman, especialmente para la economía, de lo dicho hasta aquí podrían señalarse al menos tres cuestiones relevantes para la disciplina en cuya discusión la obra hirschmaniana puede ser de gran ayuda e inspiración. Primeramente, a nivel epistémico-metodológico, la cuestión teoría-realidad y sus consecuencias metodológicas. En segundo lugar, el carácter de los vínculos entre diversas disciplinas científicas. En tercer lugar, la relación entre teoría y práctica en el marco de la economía como ciencia social. El carácter decididamente realista del enfoque hirschmaniano, su constante tendencia a -y modo de - "atravesar fronteras" disciplinarias (1999, 83; Santiso, 2000), y su clara consciencia de las implicancias ético-políticas de los estudios económico-sociales, son elementos que interpelan a la economía contemporánea aportando ideas de gran riqueza para, al menos -y parafraseando al autor- " "elevar el nivel de debate".

\section{Referencias bibliográficas}

Adelman, Jeremy. 2016. In Praise of Small: Albert O. Hirschman and the Question of Scale. Research in the History of Economic Thought and Methodology, Volume 34B, 41-61.

Adelman, Jeremy. 2013a. Worldly Philosopher: The Odyssey of Albert O. Hirschman. Princeton: Princeton University Press.

Adelman, Jeremy. 2013b. Albert O. Hirschman, un sabio. Revista de Economía Institucional, Vol. 15, 28, 13-18.

Adelman, Jeremy. 2008. Observando a Colombia: Albert O. Hirschman y la Economía del Desarrollo. Desarrollo y sociedad, 62, 1-37.

Alacevich, Michele. 2010. La economía política del Banco Mundial. Los primeros años. Colombia: Banco Mundial y Mayol Ediciones

Alacevich, Michele. 2016. Albert O. Hirschman and the Rise and Decline of Development Economics. Research in the History of Economic Thought and Methodology, Volume 34B, 13-39.

Alacevich, Michele. 2017a. Albert Hirschman, Development Economics, and the Social Sciences. En First Conference on Albert Hirschman's Legacy: Theory and Practice, Boston University, Pardee School of Global Studies.

Alacevich, Michele. 2017b. Albert Hirschman's approach of observation in "Development Projects Observed". En L. Meldolesi y N. Stame (eds.), For a Better World. Excerpts from the First Conference on Albert Hirschman's Legacy: Theory and Practice. Roma, IDE, 75-82.

Blanco, Luis Armando. 2013. Hirschman: Un gran científico social. Revista de Economía Institucional, Vol. 15, 28, 47-64.

Boianovsky, Mauro. 2015. Beyond Capital Fundamentalism: Harrod, Domar and the History of Development Economics. CHOPE Working Paper, 2015-12.

Bustelo, Pablo. 1998. Teorías contemporáneas del desarrollo económico. Madrid, Síntesis.

Caballero Argáez, Carlos. 2008. Albert Hirschman en Colombia y la planeación del desarrollo. Desarrollo y Sociedad, 62, 165-202. 
De Haan, Peter. 2016. From Keynes to Piketty. The Century that Shook Up Economics. London, Palgrave Macmillan.

Feinstein, Osvaldo. 2006. Hirschman, Albert Otto (b. 1915). En Clark, A. David (ed.), The Elgar Companion to Development Studies, Cheltenham, Edward Elgar, 226-230.

Feinstein, Osvaldo. 2017. A Hirschmanian approach to development evaluation. En L. Meldolesi y N. Stame (eds.), For a Better World. Excerpts from the First Conference on Albert Hirschman's Legacy: Theory and Practice, Roma, IDE, 104-107.

Flyvbjerg, Bent (2016). The Fallacy of Beneficial Ignorance: A Test of Hirschman's Hiding Hand, World Development, Vol. 84, 176-189.

Furió-Blasco, Elies (ed.). 1998. Albert O. Hirschman y el camino hacia el desarrollo económico. Una antología de ensayos anteriores a "La estrategia del desarrollo económico". México, FCE.

Furió-Blasco, Elies. 1996. Enlaces, estímulos y plasticidad. Un recorrido por la obra de Albert O. Hirschman. El trimestre económico, 252, 1241-1276.

Ginzburg, Andrea. 2016. The Contemporary Significance of a Dissenter: Albert O. Hirschman's Idea of Development. Research in the History of Economic Thought and Methodology, Volume 34B, 63-90.

Hargreaves Heap, S.P. 2016. "Good and Bad" (not "Good or Bad"): Albert O. Hirschman as a Behavioral Economist. Research in the History of Economic Thought and Methodology, Volume 34B, 161-174.

Hirschman, Albert O. 1958. The Strategy of Economic Development. New Haven, Yale University Press.

Hirschman, Albert O. [1958] 1961. La estrategia del desarrollo económico. México, FCE.

Hirschman, Albert O. 1961b. Ideologies of Economic Development in Latin America. En A. O. Hirschman (ed.), Latin American Issues. Essays and Comments, New York, The Twentieth Century Fund, 3-42.

Hirschman, Albert O. [1963] 1964. Estudios sobre política económica en América Latina (En ruta hacia el progreso). Madrid, Aguilar.

Hirschman, Albert O. [1967] 1969. El comportamiento de los proyectos de desarrollo. México, Siglo XXI. Hirschman, Albert O. [1971] 1973. Desarrollo y América Latina. Obstinación por la esperanza. México, FCE.

Hirschman, Albert O. [1977] 1978. Las pasiones y los intereses. Argumentos políticos en favor del capitalismo antes de su triunfo. México, FCE.

Hirschman, Albert O. [1981] 1984. De la economía a la política y más allá. Ensayos de penetración y superación de fronteras. México, FCE.

Hirschman, Albert O. 1986a. Confesión de un disidente: nueva visita a la "estrategia del desarrollo económico". En G. Meier y D. Seers (eds.), Pioneros del desarrollo, Washington, World Bank, 94-117.

Hirschman, Albert O. [1984] 1986b. El avance en colectividad. Experimentos populares en la América Latina. México, FCE.

Hirschman, Albert O. [1982] 1986c. Interés privado y acción pública. México, FCE.

Hirschman, Albert O. 1999. A través de las fronteras. Los lugares y las ideas en el trascurso de una vida. Buenos Aires, FCE.

Hirschman, Albert O. [1991] 2001. Retóricas de la intransigencia. México, FCE.

Hirschman, Albert O. [1970] 2012. Salida, voz y lealtad. Respuestas al deterioro de empresas, organizaciones y estados. México, FCE.

Hirschman, Albert O. [1985] 2013. Contra la simplicidad: Tres maneras fáciles de complicar algunas categorías del discurso económico. Revista de Economía Crítica, 15, 157-176.

Hurtado, Jimena. 2014. Albert O. Hirschman y la economía del desarrollo: Lecciones para el presente. Cuadernos de Economía, 33(62), 7-31.

Kahneman, Daniel. 2012. Pensar rápido, pensar despacio. Buenos Aires, Debate Editorial.

Lepenies, Philipp H. 2008. Possibilism: An Approach to Problem-Solving Derived from the Life and Work of Albert O. Hirschman. Development and Change, 39(3), 437-459.

Meier, Gerald M. y Seers, Dudley (eds.). 1986. Pioneros del desarrollo. Washington, World Bank.

Meldolesi, Luca. 2017. Albert Hirschman, entre Europa y América Latina. Reflexiones teórico-prácticas desde una perspectiva colorniano-hirschmaniana. Bernal, UNQ.

Meldolesi, Luca. 1997. En búsqueda de lo posible. El sorprendente mundo de Albert O. Hirschman. México, FCE.

Meldolesi, L. y Stame, N. (eds.). 2017. For a Better World. Excerpts from the First Conference on Albert Hirschman's Legacy: Theory and Practice. Roma, IDE.

Ocampo, José Antonio. 2008. Hirschman, la industrialización y la teoría del desarrollo, Desarrollo y Sociedad, 62, 41-61. 
Rodrik, Dani. 2016. Las leyes de la economía. Aciertos y errores de una ciencia en entredicho. Barcelona, Deusto.

Sandilands, Roger. 2015. La misión del Banco Mundial a Colombia de 1949, y las visiones opuestas de Lauchlin Currie y Albert Hirschman. Revista de Economía Institucional, Vol. 17, 32, 213-232.

Santiso, Javier. 2000. La mirada de Hirschman sobre el desarrollo o el arte de los traspasos y las autosubversiones. Revista de la CEPAL, 70, 91-106.

Sen, Amartya. 1997. Foreword. En A. O. Hirschman, The Passions and the Interest, Princeton, Princeton University Press, ix-xix.

Shackle, G.L.S. 1976. Epistémica y economía. Crítica de las Doctrinas Económicas. México, FCE.

Sunstein, C.R. 2015. Foreword, Albert Hirschman's Hiding Hand. En A.O. Hirschman, Development Projects Observed, Washington, Brookings Institution, vii-xiii.

Thaler, Richard H. 2017. Portarse mal. El comportamiento irracional en la vida económica. Buenos Aires, Paidós.

Valencia Agudelo, Germán D. 2013. La cuestión metodológica en Albert Hirschman. Ensayos de economía, $42,223-238$. 